\title{
Cystic fibrosis gene mutations: evaluation and assessment of disease severity
}

\author{
This article was published in the following Dove Press journal: \\ Advances in Genomics and Genetics \\ 21 October 2014 \\ Number of times this article has been viewed
}

\section{Emilie Vallières \\ Joseph Stuart Elborn}

Cystic Fibrosis and Airways

Microbiology Research Group,

Queens University Belfast, Belfast, UK

\begin{abstract}
The cystic fibrosis transmembrane regulator (CFTR) gene encodes an ion channel transporter, the CFTR protein. Since its identification in 1989, more than 1,900 sequence variants have been reported, resulting in a wide spectrum of clinical phenotypes. Cystic fibrosis (CF) is associated with many CFTR mutants and there is a continuum of disease severity observed. Recent advances in fundamental research have increased our understanding of the consequent molecular defect arising from CF mutations. This knowledge has resulted in the development of $\mathrm{CF}$-specific therapies, targeting either the genetic or the molecular defect. CF care, previously focused on symptom control, is therefore moving toward a "stratified" or "precision" therapeutic approach. This review outlines normal CFTR physiology, the proposed pathologic mechanism underlying $\mathrm{CF}$ associated-lung injury, classification of CF mutations, and the CF-specific therapies recently approved or in clinical trials.
\end{abstract}

Keywords: cystic fibrosis, gene mutations, disease severity, evaluation, assessment

\section{Introduction}

Cystic fibrosis (CF) is an autosomal recessive disorder caused by mutations in the cystic fibrosis transmembrane regulator (CFTR) gene, located on the long arm of chromosome 7. The first clinical description of the syndrome occurred in 1939 and the causative gene was successfully cloned in $1989 .{ }^{1}$ Since then, significant progress has been made to better understand the underlying molecular abnormalities. Meanwhile, efficient multidisciplinary approaches tailored to optimize the care of CF patients have significantly contributed to improve the life expectancy of people with the disease. The first CF stratified therapy, the potentiator molecule ivacaftor, has recently been licensed for patients with Gly551Asp and a range of other gating mutations. This breakthrough therapy dramatically improves functional and quality of life measures, demonstrating that CFTR is a druggable target. This has raised expectations about CF-specific therapeutic expansion in the near future. Analogous to polyallelic disorders encountered in oncology or neurology, CF care is increasingly shifting from a symptomatic and reactive approach toward a more specific, precise, and personalized strategy.

\section{Physiology of the CFTR protein}

The CFTR protein is a adenosine triphosphate (ATP)-binding cassette (ABC) transporter. ${ }^{2}$ Proteins in the ABC family collectively hydrolyze ATP to transport substrates such as ions, amino acids, sugars, drugs, and proteins against a concentration gradient. ${ }^{3}$ The CFTR protein primarily transports chloride and bicarbonate ions. Their transport unit is composed of two membrane-spanning domains and two nucleotide-binding domains. ${ }^{3}$ The
Queen's University Belfast, School of Medicine, Dentistry and Biomedical Sciences, Health Sciences Building, 97 Lisburn Road, Belfast, BT9 7BL, UK Tel +442890975876

Fax +44289097 267I

Email evallieres0I@qub.ac.uk 
transport cycle begins when the appropriate substrate binds to its specific site on the membrane-spanning domains. This step promotes ligation of ATP to the nucleotide-binding domains and their subsequent dimerization. This ATP-dependent step then provides energy to release the bound substrate across the cellular membrane. Once ATP is hydrolyzed, the nucleotidebinding domain dimer is destabilized, inorganic phosphate and adenosine diphosphate (ADP) are released, and the protein regains its basal conformational state. This cycle is referred to as the ATP switch model. ${ }^{4}$

In comparison with other ABC transporters, CFTR possesses distinctive characteristics. First, it is the only ABC protein able to transport ions across the cellular membrane. Moreover, its structure encompasses a unique regulatory domain and two terminal ( $\mathrm{N}$ - and $\mathrm{C}$-) extensions..$^{5}$ These additional components are hypothesized to regulate the gating mechanism and to modulate interactions between the ion channel and other cellular proteins (Figure 1). ${ }^{6,7}$

The CFTR protein is located in the apical surface of airway, intestinal, and exocrine epithelial cells. This anion channel secretes chloride and bicarbonate into the lumen and modulates reabsorption of sodium and water by the epithelial sodium channel. ${ }^{8}$ In the lungs, functional CFTR protein ensures optimal volume, electrolyte composition, and $\mathrm{pH}$ of the airway surface liquid (ASL), a thin fluid layer protecting the epithelium from inspired air. ASL homeostasis is essential for efficient mucociliary clearance of aerial pathogens and therefore represents the first line of defense in the airways. ${ }^{9-11}$ Moreover, ASL contains
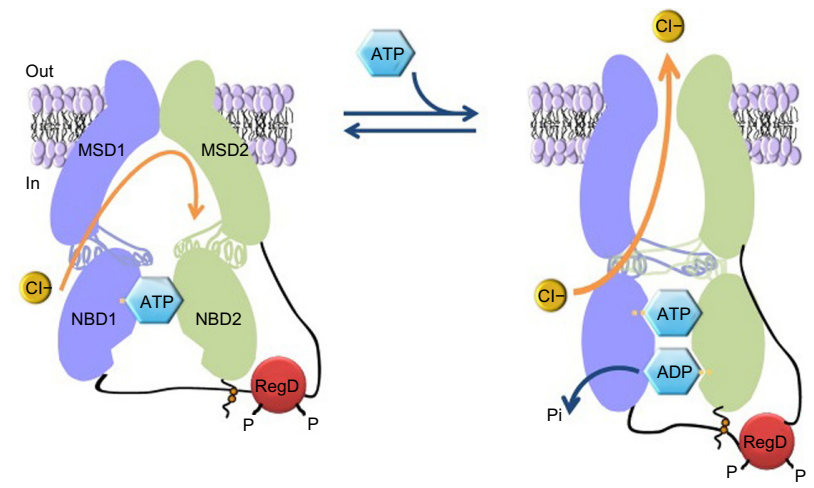

Figure I Conformational changes in the CFTR $\mathrm{Cl}^{-}$channel during channel gating. The simplified model shows a CFTR $\mathrm{Cl}^{-}$channel under quiescent (left) and activated (right) conditions.

Notes: Copyright (C) 2006. S. Karger AG, Basel. Reproduced from Chen J-H, Cai Z, Li H, Sheppard DN. Cystic Fibrosis in the 2 Ist Century. Volume 34. Basel, Switzerland: Karger; 2006. ${ }^{85}$ In and Out denote the intracellular and extracellular sides of the membrane, respectively.

Abbreviations: ADP, adenosine diphosphate; ATP, adenosine triphosphate; CFTR, cystic fibrosis transmembrane regulator; MSD, membrane-spanning domain; NBD, nucleotide-binding domain; $\mathrm{P}$, phosphorylation of RegD; Pi, inorganic phosphate; PKA, cAMP-dependent protein kinase; PPase, protein phosphatase; RegD, regulatory domain. antimicrobial peptides and phagocytic cells, which further enhance its role in the complex respiratory innate immune system.

\section{Pathophysiology underlying CF lung disease}

Chronic pulmonary disease leading to progressive decline in lung function is the chief cause of the morbidity and premature mortality observed in the CF population. Despite major advances in basic CF knowledge, the pathophysiologic process underlying CF lung disease is still a matter of some debate. Nonetheless, the low-volume hypothesis is the prevailing paradigm. ${ }^{10,12}$ Consequent to insufficient or dysfunctional CFTR protein in the airway epithelium, chloride secretion and epithelial sodium channel regulation are impaired. The endpoint is a reduction in anion secretion combined with increased sodium and water reabsorption from the ASL through the apical cellular membrane (Figure 2). This situation augments ASL viscosity and compromises mucociliary clearance efficiency, the first line of defense in the respiratory system against pathogens. Concomitantly, insufficient $\mathrm{HCO}_{3}$-secretion lowers ASL pH. ${ }^{13}$ Acidic conditions have been shown to alter bacterial killing ${ }^{14}$ by reducing the activity of ASL antimicrobial peptides ${ }^{15,16}$ and ciliary movement frequency ${ }^{17}$ while activating deleterious proteases. ${ }^{18}$ Overall, impaired lung innate immunity predisposes to pathogen colonization, which subsequently triggers neutrophil recruitment and proinflammatory cytokine secretion. This attempt by the immune system is in most cases unsuccessful and leads to a deleterious cycle of persistent infection combined with deregulated inflammation. Tissue destruction ensues and results clinically in chronic respiratory infection, lung function decline, and ultimately, respiratory failure.

\section{CF, CFTR-related disorders, and CFTR-related metabolic syndrome}

More than two decades after the description of the CF causative gene, the diagnosis of this disorder still primarily relies on clinical findings. ${ }^{19}$ The majority of CF patients fulfill the proposed diagnostic criteria, namely, one or more phenotypic characteristics associated with $\mathrm{CF}$ and a sweat chloride concentration $>60 \mathrm{mmol} / \mathrm{L}$, so are easily identified. CF phenotypic traits include chronic sinopulmonary disease, specific gastrointestinal or nutritional abnormalities, salt loss syndromes, and obstructive azoospermia. These patients are considered to have classic or typical CF, can be either pancreatic sufficient or insufficient and may display a fulminant 
A

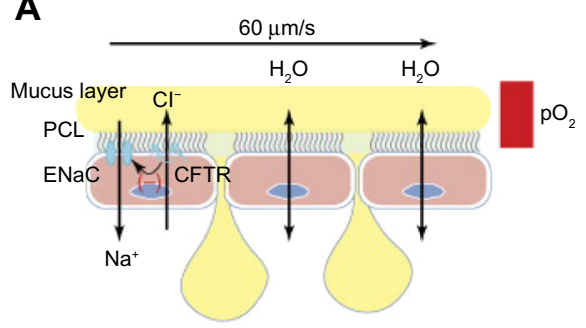

B

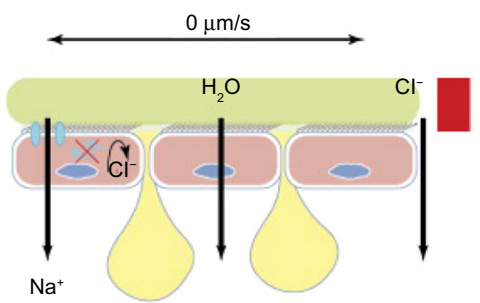

Figure 2 Normal mucus clearance mechanisms and failure of CF mucus clearance due to airway surface dehydration.

Notes: (A) Normal airways coordinate rates of $\mathrm{Na}^{+}$absorption and $\mathrm{Cl}^{-}$secretion to hydrate airway surfaces and promote mucus clearance. Water moves in response

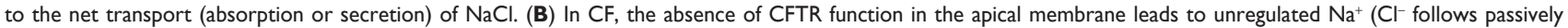
through the paracellular path; not shown), a failure to secrete $\mathrm{Cl}^{-}$and, hence, inappropriate water absorption. The dehydration of $\mathrm{CF}$ airway surfaces depletes the PCL of volume, concentrates mucus, and produces adhesion of mucus to CF airway surfaces, and hence a failure of both ciliary and cough-dependent mucus clearance. Reprinted from Trends in Molecular Medicine, I3(6), Boucher RC, Cystic fibrosis: a disease of vulnerability to airway surface dehydration, 23 I-240, Copyright (C) 2007, with permission from Elsevier. ${ }^{12}$

Abbreviations: CF, cystic fibrosis; CFTR, cystic fibrosis transmembrane regulator; ENaC, epithelial sodium channel; PCL, periciliary layer.

or protracted clinical course. ${ }^{19}$ A minority of patients, with non-classic or atypical $\mathrm{CF}$, have symptoms characteristic of $\mathrm{CF}$ in at least one organ but a negative $(<30 \mathrm{mmol} / \mathrm{L})$ or borderline (30-60 mmol/L) sweat test. Their diagnosis is confirmed by identification of CF-causing mutations in each CFTR gene or by abnormal nasal potential difference measurement. Most of these patients are pancreatic sufficient and exhibit milder lung disease. Intestinal current measurement using freshly collected rectal biopsies has shown some potential in categorizing borderline cases. ${ }^{20}$ Therefore, this technique may represent an additional tool to optimize the accuracy of CF diagnosis and when combined with specific therapeutic agents may assist in stratifying patients at a cellular level by predicting their clinical response.

More recently, the term CFTR-related disorders (CFTR-RD) has become widely accepted to describe a subgroup of patients with evident CFTR dysfunction but who do not fulfill the CF diagnostic criteria stated above. ${ }^{21,22}$ This term embraces three distinct clinical entities: congenital bilateral absence of the vas deferens, acute recurrent or chronic pancreatitis, and disseminated bronchiectasis, in the presence of CFTR dysfunction. Finally, another category has been proposed, CFTR-related metabolic syndrome, to encompass infants with abnormal immunoreactive trypsinogen levels, identified by newborn screening, but non-diagnostic sweat chloride or genetic analysis. ${ }^{23}$ These young patients, who are invariably asymptomatic at that point, may either remain symptom-free or develop CF or CFTR-RD later in life.

Nevertheless, labeling patients with classic, non-classic CF, CFTR-RD, or CFTR-related metabolic syndrome is not always straightforward because CFTR-related phenotypes encompass a continuum of clinical presentations. The extensive range of CFTR mutants,${ }^{24}$ their interaction with genetic modifiers, which are genes altering a disease course by modulating the expression of the causative gene, ${ }^{25}$ combined with the impact of lifestyle and environment lead to symptom diversity. Although efforts have been made to standardize CF diagnosis definitions, heterogeneity in the national patient registries persists and has recently been highlighted. ${ }^{26}$ Incomplete data or inclusion of patients who do not satisfy recognized diagnostic criteria (normal sweat test results, non-disease-causing mutations) were the main issues identified. In their conclusion, the authors reinforce the increasing importance of $\mathrm{CF}$ diagnosis and genotype accuracy in order to provide optimal CF care in this era where more mutation-specific therapies are being developed. ${ }^{26}$

More than 1,900 sequence variations have been identified in the encoding region of the CFTR gene. As previously mentioned, these mutations do not invariably cause $\mathrm{CF}$, so the appellation CFTR-RD has emerged. While a fraction of CFTR mutations seem to be benign and may represent fortuitous discovery (Table 1), a significant proportion are deleterious and cause either CFTR-RD or CF (examples of mutations using former and current nomenclature are shown in Table S1). CF causing mutations are typically subdivided into five classes, according to the molecular and functional defect engendered. ${ }^{27} \mathrm{~A}$ sixth class, including mutations reducing CFTR stability at the plasma membrane, has also been proposed but is yet poorly described (Figure 3 ). ${ }^{28}$

Classification of CF mutations is a useful but imperfect method to predict disease severity and guide counseling. As an example, pancreatic insufficiency, a clinical marker of severity with regard to nutritional status, is most commonly observed in class I-III mutations while patients with class IV-V mutations tend to have milder disease and consequently a better prognosis. ${ }^{29,30}$ 
Table I Examples of CFTR mutations with regard to their clinical consequences

\begin{tabular}{|c|c|}
\hline Mutation group & Examples \\
\hline A. CF-causing & 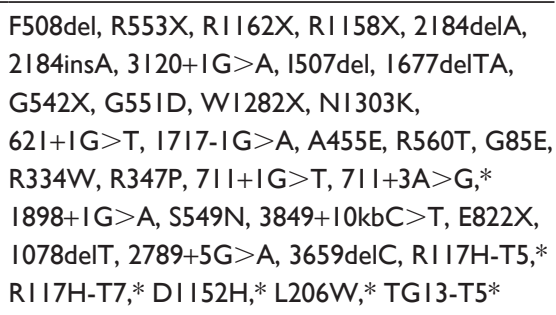 \\
\hline $\begin{array}{l}\text { B. Associated with } \\
\text { CFTR-related } \\
\text { disorders }\end{array}$ & $\begin{array}{l}\text { RII I H-T7,* TGI 2-T5,* RII I H -T5,* D I I 52H,* } \\
\text { TG I3-T5,* S997F, R297Q,* L997F, M952I, } \\
\text { D565G,* G576A,* TGII-T5,** R668C-G576A- } \\
\text { D443Y, R74W-DI270N }\end{array}$ \\
\hline $\begin{array}{l}\text { C. No clinical } \\
\text { consequences }\end{array}$ & $\begin{array}{l}\text { II48T, R75Q, 875+40A/G, M470V, E528E, } \\
\text { T854T, PI290P, 2752-I5G/C, I807M, I52IF, } \\
\text { F508C, I506V, TGII-T5** }\end{array}$ \\
\hline $\begin{array}{l}\text { D. Unknown or } \\
\text { uncertain clinical } \\
\text { relevance }\end{array}$ & Mainly missense mutations*** \\
\hline
\end{tabular}

Notes: *Mutations that may belong to either group A or group B; **mutations that may belong to either group B or group C; ***certain common sequence (missense) variants with subclinical molecular consequences (eg, M470V) that may cosegregate on the same chromosome and exert a more potent, cumulative phenotypic effect. Such polyvariant haplotypes could be potentially disease-causing. ${ }^{86}$ Only a fraction of mutations and patients have been characterized in detail, and with the exception of frequent mutations, only limited sample numbers have been available for the study of most mutations. Reprinted from the Journal of Cystic Fibrosis, 7(3), Castellani C, Cuppens H, Macek M Jr, et al, Consensus on the use and interpretation of cystic fibrosis mutation analysis in clinical practice, 179-196, Copyright (c) 2008, with permission from Elsevier. ${ }^{87}$

Abbreviations: CF, cystic fibrosis; CFTR, cystic fibrosis transmembrane regulator.

Despite significant effort from the $\mathrm{CF}$ research community, highlighted by the CFTR2 initiative (http://www.cftr2.org), this classification is still incomplete. The CFTR2 project is an international consortium that aims to define whether specific CFTR mutants can cause CF, and if so, to provide details about their pathogenic role. ${ }^{31}$ CFTR2 work is ongoing, and to date, several rare or recently identified mutations have not been sufficiently characterized to be assigned into one class. In addition, this classification scheme is not mutually exclusive, ie, a single mutation can produce a defective protein harboring characteristics of more than one class. Nevertheless, as genetic and molecular therapies are progressing into the pipeline, it is becoming crucial to obtain accurate genotypes for every $\mathrm{CF}$ patient and to promote efforts endeavoring comprehensive description of all CFTR mutants. These milestones will probably constitute sooner rather than later basic requirements in CF care.

\section{Classification of genotypes and correlation with phenotypes}

The majority of class I mutations introduce premature stop codons and are therefore non-sense mutations (eg, Arg553X,
Gly542X, Trp1282X). The derived truncated and unstable RNA segment entirely precludes the production of functional CFTR protein. Canonical splice mutations and chromosomal deletions, also leading to complete absence of CFTR protein, account for the remaining class I mutations.

Class II CFTR mutants are the most frequently encountered. Although variation is observed in different ethnic groups, approximately $90 \%$ of European and American CF patients harbor a class II mutation on at least one CFTR allele, Phe508del accounting for the vast majority of them..$^{32,33}$ In this specific example, a three-base pair deletion results in a missing amino acid, subsequent CFTR protein misfolding, and abrogation of trafficking to the cellular membrane. The faulty protein is recognized by the cellular machinery as abnormal and is degraded before it reaches the plasma membrane. Other frequent class II mutations have been identified (Gly85Glu, Arg560Thr, Ile507del, Asn1303Lys) and most of them prevent efficient trafficking. In this class, an insufficient amount of CFTR reaches the apical cell surface.

Class III mutations are characterized by deregulated opening function of an otherwise structurally normal CFTR protein. These are called gating mutations. Reduced opening time of the ion channel gate does not allow sufficient passage of chloride anions. The commonest class III mutation is Gly551Asp, the primary target of the recently released ivacaftor therapy.

Similar to class III mutations, class IV comprises structurally normal CFTR with defective conductance capacity. Even when the channel is open, inefficient electrolyte transport occurs. Arg117His and Arg347Pro belong to this group.

In classes V and VI, a reduced amount of intact CFTR proteins are present in the cell membrane. Class V comprises intron mutations, which affect splicing and reduce CFTR synthesis. Overall CFTR function is deficient due to the paucity of protein at the cellular surface but each individual protein exhibits normal function. Finally, class VI missense mutations (eg, 432delTC) result in an unstable protein. Increased turnover due to a shortened protein halflife decreases the quantity of functional CFTR present at the plasma surface.

To further complicate the classification process, some CFTR alleles encompass two distinct mutations, referred to as complex alleles. The resultant effect is difficult to predict, with an additive impact being described in specific cases. ${ }^{34}$ Moreover, some mutations were shown to impair more than a single process. As an example, the most frequent $\mathrm{CF}$ 


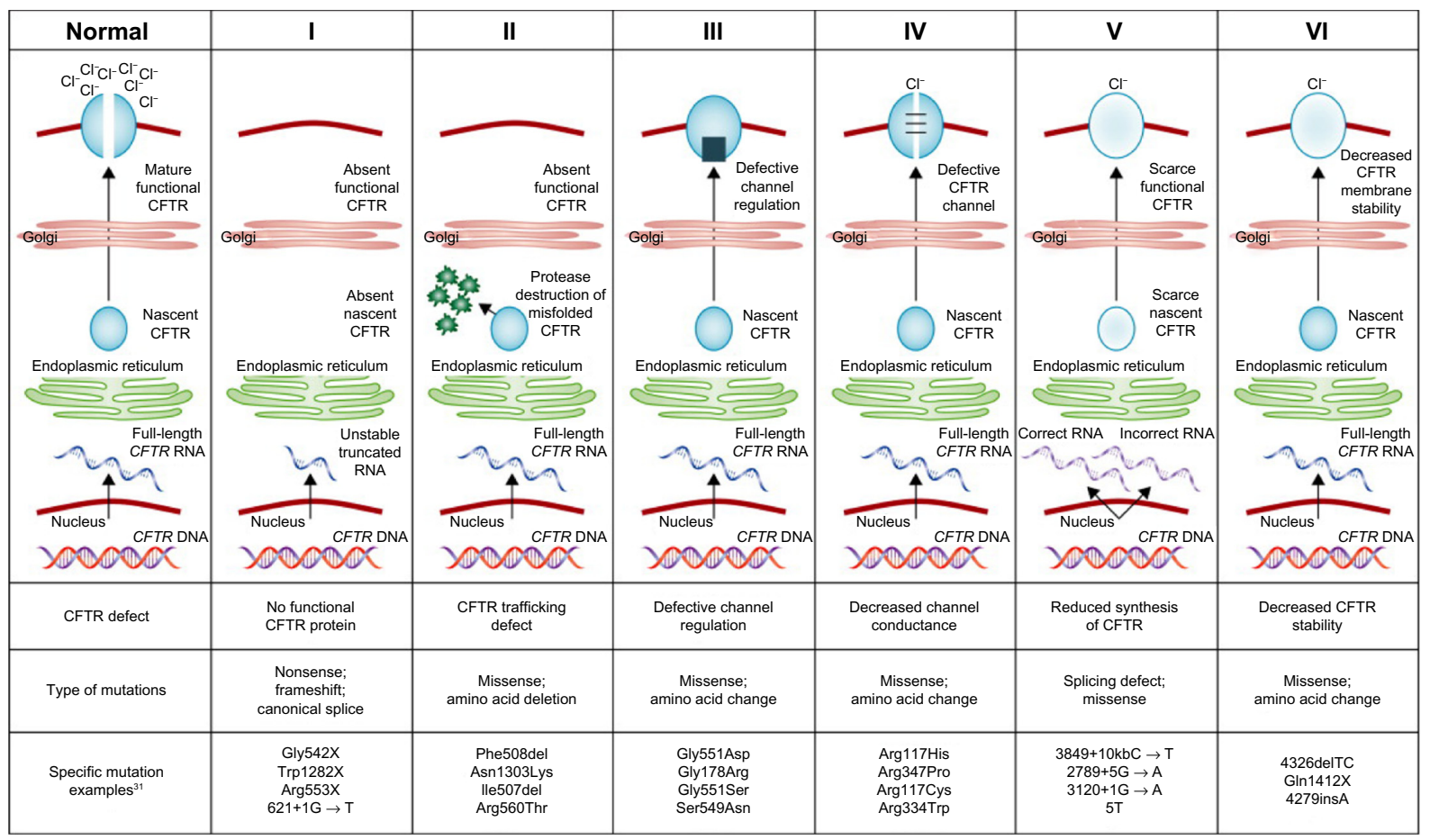

Figure 3 Classes of CFTR mutations.

Note: Reprinted from The Lancet Respiratory Medicine, I (2), Boyle MP, De Boeck K. A new era in the treatment of cystic fibrosis: correction of the underlying CFTR defect, 158-163, Copyright (c) 2013, with permission from Elsevier. ${ }^{88}$

Abbreviation: CFTR, cystic fibrosis transmembrane regulator.

mutation, Phe508del, also creates a gating defect (class III) amongst the few misfolded CFTR proteins that successfully escape the proteasome pathway and reach the apical cellular membrane. ${ }^{35}$ This situation underlies how crucial it is to adequately associate genetic and molecular defects and illustrates the expected requisite for combination therapy.

\section{Stratified treatment in CF}

Guidelines defining CF "standard of care" mostly contain therapeutic recommendations targeting symptom management rather than the fundamental cellular defect. ${ }^{36-38}$ Mucolytic, hydrating, and bronchodilator agents are used to facilitate lung mucus clearance, antibiotics are prescribed to control chronic respiratory infections or to treat acute episodes of infective exacerbations, and anti-inflammatory drugs are used to reduce the deleterious inflammatory reaction. Pancreatic enzymes and vitamin supplements are prescribed for patients who are pancreatic insufficient in order to optimize their nutritional status. These are only a few examples of the reactive strategy that used to prevail in CF care. However, recent clinical studies brought the hope that an extended range of molecules, interacting at the gene or molecular level, will soon be available to impede development of symptoms, prevent clinical deterioration, and even restore patient quality of life.
Therefore, as CF-specific drugs are now a reality, understanding molecular defects associated with specific CFTR mutation is essential to ensure optimal CF care. CF-specific therapies can be stratified in three broad categories, ie, potentiator (modulator) drugs, corrector molecules, and gene therapy.

\section{Corrector and potentiator molecules}

Apart from gene therapy-associated components, CF drugs under development can be defined as either correctors or potentiators. As their names suggest, the former group aims to repair the molecular defect (eg, a folding defect in Phe508del) while the latter group enhances channel activity without correcting the basic genetic or structural abnormality. Drugs from each category are described in the "Ivacaftor" section.

\section{Ivacaftor}

When the CF gene was identified in 1989, the CF community expected this discovery to rapidly lead to therapies targeting the molecular abnormality. However, more than 20 years passed before commercialization of the first CFTR potentiator, ivacaftor (formerly known as VX-770; Vertex Pharmaceuticals Inc., Boston, MA, USA). The 
pioneer study evaluating the efficacy and safety of ivacaftor recruited CF patients, 12 years of age or older, carrying at least one Gly551Asp allele and presenting with a forced expiratory volume in one second $\left(\mathrm{FEV}_{1}\right)$ between $40 \%$ and $90 \%$ of the predicted value. ${ }^{39}$ Significant, rapid, and sustained improvement in pulmonary function was noted in the ivacaftor group, in addition to a reduced number of pulmonary exacerbations. ENVISION (ClinicalTrials.gov identifier NCT00909727), a similar clinical trial that enrolled pediatric patients aged $6-11$ years, ${ }^{40}$ detected a significantly greater increase in $\mathrm{FEV}_{1}$ and weight gain in the treatment group compared with the placebo group. These groundbreaking results accelerated the ivacaftor approval process by the European Medicines Agency and its American counterpart, the US Food and Drug Administration. A further observational study assessed ivacaftor therapy efficacy amongst CF patients with Gly551Asp mutation and severe lung disease ( $\mathrm{FEV}_{1}<40 \%$ or on transplant waiting list), a subgroup excluded from the initial Phase III trial. ${ }^{41}$ These patients, who received ivacaftor after enrolment on a compassionate use program, demonstrated significant lung function and weight improvement and required fewer intravenous antibiotic days, similarly to the patients enrolled in the original study.

Ivacaftor increases the CFTR channel opening probability and consequently restores electrolyte exchange and mucus hydration pathways. Interestingly, its activity has been demonstrated in wild-type channel and several distinct mutants, including Phe508del. ${ }^{42,43}$ These promising in vitro data ${ }^{42}$ supported the early progress to Phase III clinical trials (ClinicalTrials.gov identifiers NCT01614470 and NCT01707290) to determine the efficacy of ivacaftor in non-Gly551Asp class III patients. In February 2014, the US Food and Drug Administration approved a supplemental New Drug Application for ivacaftor, extending its use to patients with non-Gly551Asp class III mutations. This decision was based on data released from the recent Phase III trials. Other encouraging preclinical studies suggested that ivacaftor might also be beneficial to patients with the Arg117His class IV mutation. ${ }^{44}$ However, KONDUCT (ClinicalTrials.gov identifier NCT01614457), a Phase III, randomized, placebo-controlled trial that evaluated the efficacy of ivacaftor amongst CF patients 6 years of age and older with the Arg117His mutation, did not meet its primary endpoint, ie, a significant increase in $\mathrm{FEV}_{1} \cdot{ }^{45}$ Nonetheless, subsequent subgroup analyses suggest that this drug might be useful for patients older than 18 years who have lower baseline lung function in comparison with pediatric patients.
Classes III and IV mutations are present in a minority of CF patients, and the above results will not substantially expand the proportion of patients eligible for ivacaftor therapy. As a result, extensive efforts are dedicated to develop drugs that would be efficient in more frequent CF mutants, predominantly in classes I and II. Unfortunately, the intrinsic molecular defect of these mutants constitutes the biggest challenge facing CF researchers; either no protein is made (class I) or the proteins produced are prematurely degraded (class II). Consequently, corrector molecules, in combination with potentiator molecules, will most likely be required in these situations.

\section{Potential corrector drugs}

\section{Class I: promoting translational read through}

A strategy to overcome premature termination codons (PTC) in class I mutations is to reduce ribosomal translational fidelity. PTC suppressors, such as aminoglycosides, can bind to the ribosome and alter recognition of the PTC. This action simultaneously triggers recruitment of a tRNA molecule rather than a termination complex. This tactic, which restores PTC into sense codons, allows ribosomes to read through the pathogenic stop codon without interrupting protein synthesis. A sufficient quantity of full-length CFTR proteins may then be produced to regain a non-pathologic chloride secretion level. This approach may improve clinical outcomes, as previously suggested by both in vitro and in vivo studies. ${ }^{46,47}$

Recently, ataluren (formerly known as PTC124; PTC Therapeutics, South Plainfield, NJ, USA), a non-antibiotic, non-nephrotoxic, non-ototoxic, and orally bioavailable PTC suppressor, has been evaluated. Phase I and II clinical trial results were promising, showing substantial improvement in nasal potential difference measurement. ${ }^{48-51}$ Unfortunately, a randomized, double-blind, placebo-controlled Phase III trial failed to reach its primary endpoint of a significant increase in $\mathrm{FEV}_{1}$. Secondary statistical analysis suggested that the lack of efficacy observed in the latter study might be explained by a medical interaction in patients concomitantly treated with inhaled tobramycin. ${ }^{52}$ Therefore, a second Phase III trial (ClinicalTrials.gov identifier NCT02139306) is being initiated and is recruiting $\mathrm{CF}$ patients who do not receive chronic inhaled aminoglycosides. Moreover, second-generation aminoglycosides are being developed and are expected to exhibit better read through potential and reduced toxicity when compared with their first-generation homologs. ${ }^{53}$ Novel non-aminoglycoside PTC suppressors are also in the pipeline. ${ }^{54}$ 
Furthermore, a novel strategy to counteract the PTC defect has recently been described. Amlexanox, a drug previously used to control asthma, has been shown to inhibit the non-sense mediator decay pathway while promoting read through. ${ }^{55}$ This dual mechanism of action might be a valuable approach in genetic disorders caused by PTC.

\section{Class II: pharmacologic chaperones and proteostasis regulators}

Class II mutations, including Phe508del, create a CFTR conformational abnormality that prevents adequate folding. The protein is rapidly recognized by the endoplasmic reticulum quality control mechanism and degraded before it can reach its target site, the apical cellular surface. Two strategies have been pursued to counteract this defect. Pharmacologic chaperones are small molecules that are able to bind the CFTR channel and cause stabilization of the protein, while proteostasis regulators may block degradation of the partially folded protein at the endoplasmic reticulum or plasma membrane levels.

Using high-throughput screening, some promising compounds with corrector activity have been identified. ${ }^{56-58}$ Although most of them are still in a preliminary development stage, in vitro data suggest that their success will be maximized if given in association with a potentiator in order to address coexisting defects, such as the gating anomaly in Phe508del mutants. ${ }^{59}$

Amongst them, lumacaftor (VX-809; Vertex Pharmaceuticals, Cambridge, MA, USA), a pharmacologic chaperone, has been shown to restore chloride secretion in Phe508delCFTR mutant cells to a level approaching $15 \%$ of non-CF human bronchial epithelial cells. ${ }^{59}$ Two Phase III clinical trials (TRAFFIC and TRANSPORT; ClinicalTrials.gov identifiers NCT01807923 and NCT01807949, respectively) studying the efficacy and safety of lumacaftor combined with ivacaftor in homozygous Phe508del patients older than 12 years old are now completed. The results, which have recently been published by Vertex Pharmaceuticals Inc. in a press release, demonstrate that patients who received lumacaftor in combination with ivacaftor experienced a small but statistically significant increase in $\mathrm{FEV}_{1}$ and a useful reduction in rate of pulmonary exacerbations when compared with the placebo group. ${ }^{60}$ These results are concordant with the previous Phase II study, which detected a significant $\mathrm{FEV}_{1}$ improvement in patients treated with the combination therapy. ${ }^{61}$ However, two recent in vitro studies demonstrated that long-term administration of ivacaftor destabilizes Phe508del-CFTR protein and increases its turnover rate at the plasmatic membrane. These findings suggest that the combination of lumacaftor and iva- caftor might not be optimal for patients harboring this class II mutation and might also explain the small clinical benefit observed in the previous studies. ${ }^{62,63}$ Finally, two additional Phase II trials (ClinicalTrials.gov identifiers NCT01531673 and NCT02070744) are evaluating the efficacy and safety of VX-661 (Vertex Pharmaceuticals Inc.), another CFTR corrector, alone and in combination with ivacaftor.

\section{Gene addition and correction therapy}

The molecules previously described target either the structure or the function of CFTR-mutant protein. Despite completion of several clinical trials, therapies aiming to correct the faulty gene are still awaited. ${ }^{64}$ The enormous interest devoted to gene addition therapy resides in its potential application in all CF mutation classes.

Various gene addition strategies (viral, adeno-associated virus [AAV], and non-viral vector) have been pursued for many years. Unfortunately, most of them have been unsuccessful so far, and there is actually insufficient evidence to support this type of therapy in clinical practice.$^{65}$ Nevertheless, the knowledge gained from preclinical studies attempting stable transduction with transgenes is considered to be convincing proof-of-concept. ${ }^{64} \mathrm{An}$ adenoviral vector was the initial strategy investigated. However, this vector was shown to induce potent cellular and humoral immune responses that preclude subsequent readministration. ${ }^{66}$

The AAV vector strategy has subsequently surpassed the adenoviral vectors. AAV vectors do not contain viral genes and remain episomal inside the nucleus. An initial Phase I trial indicated that AAV vectors represent safe gene transfer agents,${ }^{67}$ although a larger clinical study did not detect a significant improvement in lung function. ${ }^{68}$ The most plausible explanations underlying these results are the incapacity of the vector to efficiently transduce the airway epithelial cells in combination with stimulation of an immune response analogous to that observed with adenoviral vectors. ${ }^{69}$

Other studies using respiratory viruses have efficiently transfected airway epithelial cells, but to date none have met the criteria to progress into clinical trial. ${ }^{70,71}$ More recently, lentiviral vectors have been developed. Murine model studies demonstrated persistent gene expression and feasibility of repeated administration. ${ }^{72,73}$ Safety and production issues are currently being addressed to ensure progression of this vector into clinical studies. ${ }^{69}$

Non-viral gene transfer agents have also been produced. Cationic lipid or polymer carrier molecules bind to plasmidic DNA and promote cellular integration. Although this 
strategy is thought to be less efficient than the viral vector strategy at transfecting cells, it might escape the immune response more easily and enable repeated administration. ${ }^{74}$ Monthly aerosolized lipid vector-based gene therapy is actually being studied in a Phase IIb clinical trial (ClinicalTrials.gov identifier NCT01621867) led by the UK CF Gene Therapy Consortium. The results of this study are expected to be released in October 2014.

Furthermore, a novel approach using a recombinant enzyme to transform PTC into a non-pathogenic codon at the RNA level has recently been published. ${ }^{75}$ Within in vitro models, the authors corrected the faulty mRNA using a genetically encoded editase and restored CFTR function. A similar strategy, using antisense oligonucleotide to correct the aberrant RNA sequence, is being pursued by ProQR Therapeutics (Leiden, The Netherlands).

Barriers associated with gene therapy are being progressively and constantly overcome. Nevertheless, it remains challenging to homogeneously deliver gene transfer agents to the CF lung and accurately assess the clinical outcome of such treatment. Several obstacles remain to be resolved in order to optimize gene addition therapy, including penetration of the thick mucus layer in order to allow drug components to reach their target, ie, the airways epithelial cells.

\section{Individualized diagnosis}

Encouraged by advances realized in other medical areas (eg, oncology, neurology) and the recent approval of ivacaftor, CF care is moving towards a stratified personalized approach. Stratified medicine relies on identification of patient-specific characteristics to predict the success of a tailored therapeutic approach. ${ }^{76}$ In $\mathrm{CF}$, accurate patient genotype profiling and comprehensive gene mutation-function defect correlation are essential milestones that have to be achieved before universalization of this approach.

\section{Neonatal diagnosis}

Several countries have implemented a universal newborn CF screening program tracing the most prevalent mutations encountered in their population. ${ }^{77,78}$ It is globally accepted that early CF diagnosis and treatment improve the clinical outcome. However, until recently, existing therapeutic options were almost identical for all CF patients, regardless of their underlying genotype. Despite this limitation, newborn screening programs provide valuable information about worldwide $\mathrm{CF}$ and specific mutation incidence rate. ${ }^{79}$ Therefore, neonatal screening is indispensable to the implementation of personalized CF therapy. Unfortunately, the commercially available screening tools available at present do not detect all CFTR mutations. Consequently, accessibility to whole-genome sequencing technologies should be facilitated in order to obtain detailed genotype profiles in unresolved cases. Accuracy of genotypes should also be ensured; these data will become increasingly crucial for appropriate tailoring of treatment.

\section{Drug efficacy testing}

It has to be emphasized that the CFTR genotype is only one of several factors known to modulate the CF disease prognosis, and that the complete genetic background and various environmental factors also influence the clinical outcome. Several CF lung disease-modifying genes have already been identified and these might only represent the tip of the iceberg. ${ }^{25,80}$ Consequently, genotyping data alone are insufficient to accurately predict clinical phenotypes or disease evolution.

Heterogeneous drug responses have been reported amongst homozygous Gly551Asp patients treated with ivacaftor. As a result, assays designed to predict an individualized drug-response profile are attractive. Strategies utilizing the native epithelium of CF patients to describe the ex vivo drug-cellular response are being developed. Organoid models, the result of recent advances in stem cell technology, are one of the most promising techniques. Using three-dimensional culture techniques, rectal biopsy tissue is expanded to recreate intestinal histologic architecture. ${ }^{81}$ By reproducing the in vivo CFTR-mutant cellular pathways, this model may support functional studies anticipating the potential response of a specific patient to a given CF drug. This model has already revealed that organoids derived from specific patients, who harbor the same CFTR allele combination, may exhibit different responses when exposed to the same drug. ${ }^{82}$ Furthermore, the procedure to perform intestinal current measurement using rectal biopsies is being validated and standardized. It is expected that this technique would represent a sensitive outcome measure to assess CFTR function in future clinical trials. ${ }^{83}$ Indeed, assays able to predict individual pharmacokinetic behavior will constitute an important step toward implementation of stratified medicine in CF.

\section{Conclusion}

Although CF mutation identification is a fundamental step in stratified medicine, this information is virtually insignificant if not correlated with the intrinsic molecular defect. Considering that every $\mathrm{CF}$ patient has a unique genetic background encoding an unpredictable phenotype, only a comprehensive description of individual CF cellular 
pathways will adequately predict the efficacy of the therapeutic options. Ivacaftor is probably the first of a long list of CF-specific molecules and these drugs will only achieve their full potential if given to appropriate patients, namely those harboring the "desirable" abnormal phenotype. Potential advantages of using stratified medicine include optimization of drug efficacy, minimization of side effects, cost-effectiveness and, most importantly, significant clinical benefits to CF patients.

\section{Acknowledgments}

EV's work is supported by the Association of Medical Microbiology and Infectious Disease Canada/Pfizer postresidency fellowship and by Cystic Fibrosis Canada.

\section{Disclosure}

The authors have no relevant conflicts of interest to disclose.

\section{References}

1. Kerem B, Rommens JM, Buchanan JA, et al. Identification of the cystic fibrosis gene: genetic analysis. Science. 1989;245(4922):1073-1080.

2. Riordan JR, Rommens JM, Kerem B, et al. Identification of the cystic fibrosis gene: cloning and characterization of complementary DNA. Science. 1989;245(4922):1066-1073.

3. Higgins CF. ABC transporters: from microorganisms to man. Annu Rev Cell Biol. 1992;8:67-113.

4. Higgins CF, Linton KJ. The ATP switch model for ABC transporters. Nat Struct Mol Biol. 2004;11(10):918-926.

5. Hunt JF, Wang C, Ford RC. Cystic fibrosis transmembrane conductance regulator (ABCC7) structure. Cold Spring Harb Perspect Med. 2013;3(2):a009514.

6. Aleksandrov AA, Kota P, Aleksandrov LA, et al. Regulatory insertion removal restores maturation, stability and function of DeltaF508 CFTR. J Mol Biol. 2010;401(2):194-210.

7. Bozoky Z, Krzeminski M, Chong PA, Forman-Kay JD. Structural changes of CFTR R region upon phosphorylation: a plastic platform for intramolecular and intermolecular interactions. FEBS J. 2013;280(18): 4407-4416.

8. Livraghi-Butrico A, Kelly EJ, Wilkinson KJ, et al. Loss of CFTR function exacerbates the phenotype of $\mathrm{Na}(+)$ hyperabsorption in murine airways. Am J Physiol Lung Cell Mol Physiol. 2013;304(7):L469-L480.

9. Mall M, Grubb BR, Harkema JR, O’Neal WK, Boucher RC. Increased airway epithelial $\mathrm{Na}+$ absorption produces cystic fibrosis-like lung disease in mice. Nat Med. 2004;10(5):487-493.

10. Matsui H, Grubb BR, Tarran R, et al. Evidence for periciliary liquid layer depletion, not abnormal ion composition, in the pathogenesis of cystic fibrosis airways disease. Cell. 1998;95(7):1005-1015.

11. Knowles MR, Boucher RC. Mucus clearance as a primary innate defense mechanism for mammalian airways. J Clin Invest. 2002;109(5): 571-577.

12. Boucher RC. Cystic fibrosis: a disease of vulnerability to airway surface dehydration. Trends Mol Med. 2007;13(6):231-240.

13. Coakley RD, Grubb BR, Paradiso AM, et al. Abnormal surface liquid $\mathrm{pH}$ regulation by cultured cystic fibrosis bronchial epithelium. Proc Natl Acad Sci USA. 2003;100(26):16083-16088.

14. Pezzulo AA, Tang XX, Hoegger MJ, et al. Reduced airway surface $\mathrm{pH}$ impairs bacterial killing in the porcine cystic fibrosis lung. Nature. 2012;487(7405):109-113.
15. Johansson J, Gudmundsson GH, Rottenberg ME, Berndt KD, Agerberth B. Conformation-dependent antibacterial activity of the naturally occurring human peptide LL-37. J Biol Chem. 1998;273(6): 3718-3724.

16. Daher KA, Selsted ME, Lehrer RI. Direct inactivation of viruses by human granulocyte defensins. J Virol. 1986;60(3):1068-1074.

17. Clary-Meinesz C, Mouroux J, Cosson J, Huitorel P, Blaive B. Influence of external $\mathrm{pH}$ on ciliary beat frequency in human bronchi and bronchioles. Eur Respir J. 1998;11(2):330-333.

18. Britigan BE, Hayek MB, Doebbeling BN, Fick RB Jr. Transferrin and lactoferrin undergo proteolytic cleavage in the Pseudomonas aeruginosa-infected lungs of patients with cystic fibrosis. Infect Immun . 1993;61(12):5049-5055.

19. De Boeck K, Wilschanski M, Castellani C, et al. Cystic fibrosis: terminology and diagnostic algorithms. Thorax. 2006;61(7):627-635.

20. Derichs N, Sanz J, Von Kanel T, et al. Intestinal current measurement for diagnostic classification of patients with questionable cystic fibrosis: validation and reference data. Thorax. 2010;65(7):594-599.

21. [No authors listed]. Classification of cystic fibrosis and related disorders. J Cyst Fibros. 2002;1(1):5-8.

22. Bombieri C, Claustres M, De Boeck K, et al. Recommendations for the classification of diseases as CFTR-related disorders. J Cyst Fibros. 2011;10 Suppl 2:S86-S102.

23. Borowitz D, Parad RB, Sharp JK, et al. Cystic Fibrosis Foundation practice guidelines for the management of infants with cystic fibrosis transmembrane conductance regulator-related metabolic syndrome during the first two years of life and beyond. J Pediatr. 2009; 155(Suppl 6):S106-S116.

24. Sosnay PR, Siklosi KR, Van Goor F, et al. Defining the disease liability of variants in the cystic fibrosis transmembrane conductance regulator gene. Nat Genet. 2013;45(10):1160-1167.

25. Collaco JM, Cutting GR. Update on gene modifiers in cystic fibrosis. Curr Opin Pulm Med. 2008;14(6):559-566.

26. Thomas M, Lemonnier L, Gulmans V, et al. Is there evidence for correct diagnosis in cystic fibrosis registries? J Cyst Fibros. 2014;13(3): 275-280.

27. Welsh MJ, Smith AE. Molecular mechanisms of CFTR chloride channel dysfunction in cystic fibrosis. Cell. 1993;73(7):1251-1254.

28. Haardt M, Benharouga M, Lechardeur D, Kartner N, Lukacs GL. C-terminal truncations destabilize the cystic fibrosis transmembrane conductance regulator without impairing its biogenesis. A novel class of mutation. J Biol Chem. 1999;274(31):21873-21877.

29. McKone EF, Goss CH, Aitken ML. CFTR genotype as a predictor of prognosis in cystic fibrosis. Chest. 2006;130(5):1441-1447.

30. Koch C, Cuppens H, Rainisio M, et al. European Epidemiologic Registry of Cystic Fibrosis (ERCF): comparison of major disease manifestations between patients with different classes of mutations. Pediatr Pulmonol. 2001;31(1):1-12.

31. US Cystic Fibrosis Foundation. The Clinical and Functional Translation of CFTR. Available from: http://www.cftr2.org/. Accessed June 8, 2014

32. Cystic Fibrosis Foundation Patient Registry. Annual Data Report 2011. Bethesda, MD, USA: Cystic Fibrosis Foundation; 2013.

33. De Boeck K, Zolin A, Cuppens H, Olesen HV, Viviani L. The relative frequency of CFTR mutation classes in European patients with cystic fibrosis. J Cyst Fibros. 2014;13(4):403-409.

34. Clain J, Fritsch J, Lehmann-Che J, et al. Two mild cystic fibrosis-associated mutations result in severe cystic fibrosis when combined in cis and reveal a residue important for cystic fibrosis transmembrane conductance regulator processing and function. J Biol Chem. 2001;276(12): 9045-9049.

35. Dalemans W, Barbry P, Champigny G, et al. Altered chloride ion channel kinetics associated with the delta F508 cystic fibrosis mutation. Nature. 1991;354(6354):526-528.

36. Flume PA, Mogayzel PJ Jr, Robinson KA, et al. Cystic fibrosis pulmonary guidelines: treatment of pulmonary exacerbations. Am J Respir Crit Care Med. 2009;180(9):802-808.

37. Kerem E, Conway S, Elborn S, Heijerman H. Standards of care for patients with cystic fibrosis: a European consensus. J Cyst Fibros. 2005;4(1):7-26. 
38. Mogayzel PJ Jr, Naureckas ET, Robinson KA, et al. Cystic fibrosis pulmonary guidelines. Chronic medications for maintenance of lung health. Am J Respir Crit Care Med. 2013;187(7):680-689.

39. Ramsey BW, Davies J, McElvaney NG, et al. A CFTR potentiator in patients with cystic fibrosis and the G551D mutation. $N$ Engl J Med. 2011;365(18):1663-1672.

40. Davies JC, Wainwright CE, Canny GJ, et al. Efficacy and safety of ivacaftor in patients aged 6 to 11 years with cystic fibrosis with a G551D mutation. Am J Respir Crit Care Med. 2013;187(11):1219-1225.

41. Barry PJ, Plant BJ, Nair A, et al. Effects of ivacaftor in patients with cystic fibrosis who carry the G551D mutation and have severe lung disease. Chest. 2014;146(1):152-158.

42. Yu H, Burton B, Huang CJ, et al. Ivacaftor potentiation of multiple CFTR channels with gating mutations. J Cyst Fibros. 2012;11(3):237-245.

43. Van Goor F, Hadida S, Grootenhuis PD, et al. Rescue of CF airway epithelial cell function in vitro by a CFTR potentiator, VX-770. Proc Natl Acad Sci USA. 2009;106(44):18825-18830.

44. Char JE, Wolfe MH, Cho HJ, et al. A little CFTR goes a long way: CFTR-dependent sweat secretion from G551D and R117H-5T cystic fibrosis subjects taking ivacaftor. PLoS One. 2014;9(2):e88564.

45. Moss RB, Flume PA, Elborn JS, et al. Ivacaftor treatment in patients with cystic fibrosis who have an R117H-CFTR mutation, the KONDUCT Study. J Cyst Fibros. 2014;13(S2):S44.

46. Wilschanski M, Yahav Y, Yaacov Y, et al. Gentamicin-induced correction of CFTR function in patients with cystic fibrosis and CFTR stop mutations. N Engl J Med. 2003;349(15):1433-1441.

47. Sermet-Gaudelus I, Renouil M, Fajac A, et al. In vitro prediction of stop-codon suppression by intravenous gentamicin in patients with cystic fibrosis: a pilot study. BMC Med. 2007;5:5.

48. Wilschanski M, Miller LL, Shoseyov D, et al. Chronic ataluren (PTC124) treatment of nonsense mutation cystic fibrosis. Eur Respir J. 2011;38(1):59-69.

49. Sermet-Gaudelus I, Boeck KD, Casimir GJ, et al. Ataluren (PTC124) induces cystic fibrosis transmembrane conductance regulator protein expression and activity in children with nonsense mutation cystic fibrosis. Am J Respir Crit Care Med. 2010;182(10):1262-1272.

50. Kerem E, Hirawat S, Armoni S, et al. Effectiveness of PTC124 treatment of cystic fibrosis caused by nonsense mutations: a prospective phase II trial. Lancet. 2008;372(9640):719-727.

51. Hirawat S, Welch EM, Elfring GL, et al. Safety, tolerability, and pharmacokinetics of PTC124, a nonaminoglycoside nonsense mutation suppressor, following single- and multiple-dose administration to healthy male and female adult volunteers. J Clin Pharmacol. 2007;47(4):430-444.

52. Kerem E, Konstan MW, De Boeck K, et al. Ataluren for the treatment of nonsense-mutation cystic fibrosis: a randomised, double-blind, placebocontrolled phase 3 trial. Lancet Respir Med. 2014;2(7):539-547.

53. Rowe SM, Sloane P, Tang LP, et al. Suppression of CFTR premature termination codons and rescue of CFTR protein and function by the synthetic aminoglycoside NB54. J Mol Med (Berl). 2011;89(11): 1149-1161.

54. Du L, Damoiseaux R, Nahas S, et al. Nonaminoglycoside compounds induce readthrough of nonsense mutations. $J$ Exp Med. 2009;206(10): 2285-2297.

55. Gonzalez-Hilarion S, Beghyn T, Jia J, et al. Rescue of nonsense mutations by amlexanox in human cells. Orphanet J Rare Dis. 2012;7:58.

56. Grove DE, Rosser MF, Ren HY, Naren AP, Cyr DM. Mechanisms for rescue of correctable folding defects in CFTRDelta F508. Mol Biol Cell. 2009;20(18):4059-4069.

57. Pedemonte N, Tomati V, Sondo E, et al. Dual activity of aminoarylthiazoles on the trafficking and gating defects of the cystic fibrosis transmembrane conductance regulator chloride channel caused by cystic fibrosis mutations. J Biol Chem. 2011;286(17):15215-15226.

58. Ye L, Knapp JM, Sangwung P, Fettinger JC, Verkman AS, Kurth MJ. Pyrazolylthiazole as DeltaF508-cystic fibrosis transmembrane conductance regulator correctors with improved hydrophilicity compared to bithiazoles. J Med Chem. 2010;53(9):3772-3781.
59. Van Goor F, Hadida S, Grootenhuis PD, et al. Correction of the F508delCFTR protein processing defect in vitro by the investigational drug VX-809. Proc Natl Acad Sci USA. 2011;108(46):18843-18848.

60. [No authors listed]. Two 24-week phase 3 studies of lumacaftor in combination with ivacaftor met primary endpoint with statistically significant improvements in lung function (FEV1) in people with cystic fibrosis who have two copies of the F508del Mutation (Press release). Cambridge, MA, USA: Vertex Pharmaceuticals Inc.; 2014. Available from: http://investors.vrtx.com/releasedetail.cfm?ReleaseID=856185. Accessed August 25, 2014

61. Boyle MP, Bell SC, Konstan MW, et al. A CFTR corrector (lumacaftor) and a CFTR potentiator (ivacaftor) for treatment of patients with cystic fibrosis who have a phe508del CFTR mutation: a phase 2 randomised controlled trial. Lancet Respir Med. 2014;2(7):527-538.

62. Veit G, Avramescu RG, Perdomo D, et al. Some gating potentiators, including VX-770, diminish $\triangle$ F508-CFTR functional expression. Sci Transl Med. 2014;6(246):246ra297.

63. Cholon DM, Quinney NL, Fulcher ML, et al. Potentiator ivacaftor abrogates pharmacological correction of $\triangle \mathrm{F} 508$ CFTR in cystic fibrosis. Sci Transl Med. 2014;6(246):246ra296.

64. Griesenbach U, Alton EW. Gene transfer to the lung: lessons learned from more than 2 decades of CF gene therapy. Adv Drug Deliv Rev. 2009;61(2):128-139.

65. Lee TW, Southern KW. Topical cystic fibrosis transmembrane conductance regulator gene replacement for cystic fibrosis-related lung disease. Cochrane Database Syst Rev. 2012;10:CD005599.

66. Harvey BG, Leopold PL, Hackett NR, et al. Airway epithelial CFTR mRNA expression in cystic fibrosis patients after repetitive administration of a recombinant adenovirus. J Clin Invest. 1999;104(9): $1245-1255$.

67. Moss RB, Rodman D, Spencer LT, et al. Repeated adeno-associated virus serotype 2 aerosol-mediated cystic fibrosis transmembrane regulator gene transfer to the lungs of patients with cystic fibrosis: a multicenter, double-blind, placebo-controlled trial. Chest. 2004; 125(2):509-521.

68. Moss RB, Milla C, Colombo J, et al. Repeated aerosolized AAV-CFTR for treatment of cystic fibrosis: a randomized placebo-controlled phase $2 \mathrm{~B}$ trial. Hum Gene Ther. 2007;18(8):726-732.

69. Griesenbach U, Alton EW. Moving forward: cystic fibrosis gene therapy. Hum Mol Genet. 2013;22(R1):R52-R58.

70. Ferrari S, Griesenbach U, Shiraki-Iida T, et al. A defective nontransmissible recombinant Sendai virus mediates efficient gene transfer to airway epithelium in vivo. Gene Ther. 2004;11(22):1659-1664.

71. Zhang L, Bukreyev A, Thompson CI, et al. Infection of ciliated cells by human parainfluenza virus type 3 in an in vitro model of human airway epithelium. J Virol. 2005;79(2):1113-1124.

72. Mitomo K, Griesenbach U, Inoue M, et al. Toward gene therapy for cystic fibrosis using a lentivirus pseudotyped with Sendai virus envelopes. Mol Ther. 2010;18(6):1173-1182.

73. Sinn PL, Arias AC, Brogden KA, McCray PB Jr. Lentivirus vector can be readministered to nasal epithelia without blocking immune responses. J Virol. 2008;82(21):10684-10692.

74. Hyde SC, Southern KW, Gileadi U, et al. Repeat administration of DNA/ liposomes to the nasal epithelium of patients with cystic fibrosis. Gene Ther. 2000;7(13):1156-1165.

75. Montiel-Gonzalez MF, Vallecillo-Viejo I, Yudowski GA, Rosenthal JJ. Correction of mutations within the cystic fibrosis transmembrane conductance regulator by site-directed RNA editing. Proc Natl Acad Sci USA. 2013;110(45):18285-18290.

76. Hall IP. Stratified medicine: drugs meet genetics. Eur Respir Rev. 2013;22(127):53-57.

77. Castellani C, Southern KW, Brownlee K, et al. European best practice guidelines for cystic fibrosis neonatal screening. J Cyst Fibros. 2009;8(3):153-173.

78. Comeau AM, Accurso FJ, White TB, et al. Guidelines for implementation of cystic fibrosis newborn screening programs: Cystic Fibrosis Foundation workshop report. Pediatrics. 2007;119(2):e495-e518. 
79. Bobadilla JL, Macek M Jr, Fine JP, Farrell PM. Cystic fibrosis: a worldwide analysis of CFTR mutations correlation with incidence data and application to screening. Hum Mutat. 2002;19(6):575-606.

80. Guillot L, Beucher J, Tabary O, Le Rouzic P, Clement A, Corvol H. Lung disease modifier genes in cystic fibrosis. Int J Biochem Cell Biol. 2014;52:83-93.

81. Sato T, Clevers H. Growing self-organizing mini-guts from a single intestinal stem cell: mechanism and applications. Science. 2013; 340(6137):1190-1194.

82. Dekkers JF, Wiegerinck CL, de Jonge HR, et al. A functional CFTR assay using primary cystic fibrosis intestinal organoids. Nat Med. 2013; 19(7):939-945.

83. Clancy JP, Szczesniak RD, Ashlock MA, et al. Multicenter intestinal current measurements in rectal biopsies from $\mathrm{CF}$ and non-CF subjects to monitor CFTR function. PLoS One. 2013;8(9):e73905.
84. Wang Y, Wrennall JA, Cai Z, Li H, Sheppard DN. Understanding how cystic fibrosis mutations disrupt CFTR function: from single molecules to animal models. Int J Biochem Cell Biol. 2014;52:47-57.

85. Chen J-H, Cai Z, Li H, Sheppard DN. Cystic Fibrosis in the 21st Century. Volume 34. Basel, Switzerland: Karger; 2006.

86. Cuppens H, Lin W, Jaspers M, et al. Polyvariant mutant cystic fibrosis transmembrane conductance regulator genes. The polymorphic (Tg) $\mathrm{m}$ locus explains the partial penetrance of the T5 polymorphism as a disease mutation. J Clin Invest. 1998;101(2):487-496.

87. Castellani C, Cuppens H, Macek M Jr, et al. Consensus on the use and interpretation of cystic fibrosis mutation analysis in clinical practice. J Cyst Fibros. 2008;7(3):179-196.

88. Boyle MP, De Boeck K. A new era in the treatment of cystic fibrosis: correction of the underlying CFTR defect. Lancet Respir Med. 2013; $1(2): 158-163$. 


\section{Supplementary material}

Table SI Examples of CF mutations using former and current nomenclature

\begin{tabular}{|c|c|c|}
\hline \multirow{2}{*}{$\begin{array}{l}\text { Former } \\
\text { nomenclature }\end{array}$} & \multicolumn{2}{|c|}{ Current nomenclature } \\
\hline & DNA level & Protein level \\
\hline$\Delta \mathrm{F} 508$ & c.152I_I523delCTT & p.Phe508del \\
\hline$\Delta \mathrm{I} 507$ & c.1519_I52IdelATC & p.lle507del \\
\hline $\mathrm{RII7H}$ & c. $350 \mathrm{G}>\mathrm{A}$ & p.ArgII7His \\
\hline G542X & c. $1624 G>T$ & p.Gly542X \\
\hline G55ID & c. $1652 \mathrm{G}>\mathrm{A}$ & p.Gly55IAsp \\
\hline R553X & c. $1657 \mathrm{C}>\mathrm{T}$ & p.Arg553X \\
\hline R560T & c. $1679 \mathrm{G}>\mathrm{C}$ & p.Arg560Thr \\
\hline S549N & c. $1646 \mathrm{G}>\mathrm{A}$ & p.Ser549Asn \\
\hline WI $282 X$ & c. $3846 \mathrm{G}>\mathrm{A}$ & p.Trp1282X \\
\hline NI303K & c. $3909 \mathrm{C}>\mathrm{G}$ & p.Asn I 303Lys \\
\hline G85E & c. $254 \mathrm{G}>\mathrm{A}$ & p.Gly85Glu \\
\hline A455E & c. $1364 C>A$ & p.Ala455Glu \\
\hline R334W & c. $1000 \mathrm{C}>\mathrm{T}$ & p.Arg334Trp \\
\hline R347P & c. $1040 \mathrm{G}>\mathrm{C}$ & p.Arg347Pro \\
\hline$R I I 62 X$ & c. $3484 \mathrm{C}>\mathrm{T}$ & p.ArgII62X \\
\hline 2184 delA & c.2052delA & p.Lys684fs \\
\hline
\end{tabular}

Abbreviation: $\mathrm{CF}$, cystic fibrosis.

\section{Publish your work in this journal}

Advances in Genomics and Genetics is an international, peer reviewed, open access journal that focuses on new developments in characterizing the human and animal genome and specific gene expressions in health and disease. Particular emphasis will be given to those studies that elucidate genes, biomarkers and targets in the development of new or improved therapeutic interventions. The journal is characterized by the rapid reporting of reviews, original research, methodologies, technologies and analytics in this subject area. The manuscript management system is completely online and includes a very quick and fair peer-review system. Visit http://www.dovepress.com/ testimonials.php to read real quotes from published authors. 